\title{
Descrição de um caso de paracoccidioidomicose na forma infantojuvenil
}

\section{Description of the one paracoccidioidomycosis case in the infant teenage form}

\author{
Samara Guerra Carneiro' \\ Bianca Ribeiro Barreto ${ }^{1}$ \\ João Francesco Strapasson' \\ Tiago Turci Ribeiro' \\ Albino Moreira Torres ${ }^{2}$
}

1 Dissente do Curso de Medicina do Centro Universitário de Volta Redonda - UniFOA.
2 Docente do Curso de Medicina do Centro Universitário de Volta Redonda - UniFOA.

\section{Resumo}

A paracoccidioidomicose é uma micose sistêmica. Sua infecção ocorre pela inalação dos conídeos dispersos no ar. É uma patologia que pode acometer todo o organismo humano, contendo diversas formas de apresentação. 0 objetivo deste estudo foi relatar e discutir o caso de um paciente que apresentou a forma infanto-juvenil. Paciente do sexo masculino, 11 anos, HIV negativo, natural de Mendes - RJ, criado na zona rural até os 7 anos de idade, atualmente residindo em Volta Redonda. $\mathrm{O}$ adolescente foi admitido no hospital: hipocorado $(1+/ 4)$, presença de linfoadenopatias generalizadas, hepatoesplenomegalia e iniciou quadro de febre após o terceiro dia de internação. A sorologia de TORCH não nos mostrou resultados importantes. Foi realizada biopsia linfonodal axilar esquerda, cujo resultado histopatológico foi de doença granulomatosa difusa, com imagens fúngicas semelhantes à "roda de leme", fortificando o diagnóstico de Paracococcidiodomicose. Fez uso de Anfotericina B em ambiente hospitalar e sulfametaxazol com trimetoprim, em domicílio. A suspeita dessa enfermidade é facilitada através da associação do caráter endêmico com a história e manifestações clínicas apresentadas pelos pacientes. Esse relato teve como finalidade evidenciar a importância de se elaborar uma anamnese minuciosa, o diagnóstico e o tratamento adequado dessa patologia grave que pode ser fatal.

\section{Palavras-chave}

paracoccidioidomicose, linfonodomegalia, hepatoesplenomegalia biópsia

\begin{abstract}
Paracoccidioidomycosis is systematic Mycosis. It's infection occurs by conidia inhalation spread in the air. It is a disease that can attack the entire human organism and surfaces in multiple ways. The objective of this study was to convey and analyze the case of a patient that exhibited an infant-teenage form. Male gender patient, 11 years-old, HIV negative, born in Mendes - Rio De Janeiro $(R J)$, Brazil where he was raised in a rural area until he was 7 years old and currently resides in Volta Redonda, RJ. The adolescent was admitted to the hospital: Pallid (1+/4), dispersed Lymphadenopathies, hepatosplenomegaly and developed fever after the third day of hospitalization. The TORCH serology test revealed no relevant information. A biopsy was performed on the left axillary Lymph node which the pathological result confirmed granulomatous disease diffused, with images of fungal similar in appearance to boat steering wheel, increasing the Paracoccidioidomycosis diagnostic. Amphotericin B was used within the hospital environment and Sulfametaxazol along trimethoprim at home. The suspicion of this illness is established further through association of the endemic frame with history and clinical signs presented by patients. The purpose of this report is to express the importance of conducting comprehensive anamnesis, the diagnostic, and the proper treatment of this grave pathology that could be fatal.
\end{abstract}

\section{Keywords}

Paracoccidioidomycosis, lymph, hepatoesplenomegaly, biopsy

\section{Como você deve citar?}

CARNEIRO, Samara Guerra et al. Descrição de um caso de paracoccidioidomicose na forma infantojuvenil. Cadernos UniFOA, Volta Redonda, n. 27, p. 83-87, abr. 2015. 


\section{INTRODUÇÃO}

A paracoccidioidomicose é a micose sistêmica de maior relevância na América Latina, descrita pela primeira vez em 1908, pelo brasileiro Adolph Lutz. O primeiro caso infantil, considerado raro, foi observado em 1911, por Montenegro (TONELLI E FREIRE, 2000). É causada pelo fungo termodimórfico Paracoccidioides brasiliensis e sua infecção ocorre através da inalação dos conídeos dispersos no ar, que destes originam-se as formas leveduriformes (forma parasitária), cuja evolução vai depender da quantidade e da qualidade do conídeo inalado e da resposta imune do hospedeiro (SZTAJNBOK et al., 2012; TAVARES et al. 2012).

Doença endêmica em todo o Brasil, principalmente nas regiões sudeste, sul e centro-oeste (SZTAJNBOK et al., 2012), a paracoccidioidomicose tem como característica acometer, principalmente, pessoas do sexo masculino, entre 30 e 50 anos, que trabalham ou vivem em área rural (TONELLI E FREIRE, 2000; SZTAJNBOK et al., 2012). Essa micose é rara em crianças, sendo ambos os sexos igualmente afetados até a puberdade. Após esse período, a mulher tem o efeito protetor do estrogênio (TAVARES et al., 2012).

É uma patologia que pode se manifestar em todo o organismo humano como pele, pulmões, cavidade oral, linfonodos, aparelho digestório, ossos, adrenais, mama e sistema nervoso central (TAVARES et al., 2012). A paracoccidioidomicose pode se apresentar de diferentes formas: regressiva, progressiva (aguda/subaguda ou juvenil, crônica ou do adulto e mista) e a forma residual que nomeia as sequelas da infecção (TAVARES et al., 2012).

É descrito, a seguir, um caso de paracoccidioidomicose forma progressiva e aguda do tipo ganglionar, em um adolescente de onze anos que tem histórico de vida em área rural.

\section{RELATO DE CASO}

T.S.X, 11 anos, sexo masculino, HIV negativo, natural de Mendes-RJ, deu entrada no HMMR, no dia 27 de outubro de 2012, apresentando um "caroço" indolor e mole em região axilar esquerda. Relata ainda que observou nódulos em região cervical anterior, há cerca de 3 meses. 0 adolescente nasceu em Mendes-RJ, onde foi criado em um sítio até os 7 anos de idade. Há quatro anos, reside em Volta Redonda-RJ. Durante as férias, por varias vezes, o pai levava o adolescente e os irmãos para uma fazenda no norte de Minas Gerais.

Ao exame físico, observou-se que o adolescente estava hipocorado $(1+/ 4)$, além da presença de linfonodos das cadeias pré-auricular esquerda, axilares, cervicais anteriores e posteriores, submandibulares e inguinal esquerda aumentados de tamanho, de consistência elástica, móveis e indolores. Mediam cerca de $5 \mathrm{~cm}$ e eram acompanhados de hiperemia e crostas. Além disso, foi observado que o fígado era palpável a $2 \mathrm{~cm}$ do rebordo costal e que o baço era palpável na posição de Schuster e ainda que o espaço de Traube encontrava-se ocupado. 0 restante do exame físico não apresentou alterações. No terceiro dia de internação, o paciente iniciou febre ( $T$ axilar: $38,6 \circ \mathrm{C}$ ), mantendo o quadro por alguns dias. De acordo com a USG abdominal, o baço estava aumentado de volume, apresentando múltiplas áreas nodulares hipoecóicas e hipovascularizadas e a aorta abdominal apresentava-se normal, porém havia a presença de linfonodomegalia paraórtica difusa. A sorologia de TORCH não nos mostrou resultados importantes.

No décimo quarto dia de internação, recebemos o resultado da biópsia linfonodal axilar esquerda, cujo laudo histopatológico foi de doença granulomatosa difusa, com imagens fúngicas semelhantes 
à roda de leme, fortificando o diagnóstico de Paracococcidiodomicose. A partir desse resultado foi imediatamente prescrito Anfotericina B ( $1 \mathrm{mg} / \mathrm{kg}$ de peso), na apresentação de Anfotericina B $50 \mathrm{mg} /$ $10 \mathrm{~mL}$, sendo utilizada a dose de adulto devido ao peso do paciente (P. $38 \mathrm{Kg}$ ). Porém, o adolescente apresentou certa intolerância ao medicamento, sendo considerada a dose infantil de $25 \mathrm{mg} / \mathrm{kg}$ de Anfotericina B mais adequada para o tratamento. Ainda persistindo a intolerância, ficou decidido que a droga seria feita em dias alternados, mantendo-se a dose de $25 \mathrm{mg} / \mathrm{kg}$. 0 paciente evoluiu de maneira favorável e por volta do $27^{\circ}$ dia de internação, recebeu alta hospitalar, sendo encaminhado ao serviço ambulatorial. A família se comprometeu em levar o adolescente até o hospital, em dias alternados, para que ele pudesse receber a dose de Anfotericina $B$, até quando esse medicamento pudesse ser substituído pelo Sulfometaxazol- Trimetoprim.

\section{DISCUSSÃO}

A paracoccidioidomicose, normalmente, ocorre na fase adulta jovem e sua evolução depende da virulência do fungo, da quantidade de conídeos inalados (esporos) e da integridade do hospedeiro (PALMEIRO et al., 2005). A doença é rara na infância, mas segundo Gonçalves et al (2000), em estudo retrospectivo de revisão bibliográfica de 10 relatos de caso sobre paracoccidioidomicose infantojuvenil, o número total de casos infantis vem crescendo, pelo menos na região sudeste, provavelmente como uma consequência multifatorial. Nessas formas clínicas, existe um baixo diferencial de proporção entre os gêneros masculino e feminino, comparando-se com o apresentado na forma adulta dos pacientes com a micose, sendo mais prevalente no sexo masculino ( GONÇALVES et al., 2000).

Já em um estudo realizado no período de janeiro de 1992 a janeiro de 2002, através da revisão de prontuários do serviço de pediatria do Hospital Universitário da Universidade Federal de Juiz de Fora (HU-UFJF), foi observado maior prevalência em meninos, sendo que todos os pacientes eram provenientes da zona rural (ALVES et al., 2004).

$\mathrm{Na}$ forma aguda, predominantemente juvenil, os achados clínicos são inespecíficos. Em função disso, consideram-se como diagnósticos diferenciais as doenças que cursam com linfadenomegalia, febre, hepatoesplenomegalia, perda ponderal e lesões cutâneas, como as doenças linfoproliferativas, histoplasmose, leishimaniose, tuberculose e outras micobacterioses (TELLES, 2001). 0 nosso paciente apresentou a forma aguda da doença, sendo, portanto necessários, anamnese e exame físico dirigidos para a pesquisa de múltiplas cadeias de linfonodos e suas possíveis complicações: icterícia obstrutiva por compressão do colédoco, suboclusão ou oclusão intestinal, síndrome de compressão da veia cava, diarreia com síndrome de má absorção e ascite. Além de avaliação de múltiplas cadeias de linfonodos, pesquisa de hepatomegalia, esplenomegalia, lesões de pele, lesões osteoarticulares, sinais e sintomas adrenais (astenia, hipotensão arterial, hiperpigmentação da pele, dores abdominais) e sistema nervoso central (cefaleia, déficit motor, síndrome convulsiva, alteração de comportamento e/ou nível de consciência (SHIKANAI-YASUDA et al., 2006).

A expressão clínica fundamental da forma infantojuvenil é o grande envolvimento do sistema macrofágico-linfóide, que se exterioriza por linfoadenopatias superficiais e/ou profundas, que são mais frequentemente generalizadas (ALVES et al., 2004). Isso pode ser observado no paciente relatado, uma vez que este apresentava, na admissão, linfonodos das cadeias pré-auricular esquerda, axilares, cervicais anteriores e posteriores, submandibulares e inguinal esquerda aumentados de tamanho, de consistência elástica, móveis e indolores.

$\mathrm{Na}$ literatura, evidencia-se a importância da biópsia de lesão acessível (cutânea, mucosa ou linfonodo superficial), sendo o exame microscópico direto do material obtido(análise do esfregaço ou 
material biológico diretamente em lâmina e lamínula e a cultura) preferencial, tanto pela sua acurácia quanto por sua simplicidade (TOLEDO et al, 2011; MINISTÉRIO DA SAÚDE, 2013).

Como resultado para confirmação diagnóstica, encontra-se o fungo em seu aspecto característico: roda de leme" (MINISTÉRIO DA SAÚDE, 2013). No caso apresentado, foi realizada biópsia de linfonodo axilar esquerdo, apresentando um resultado semelhante ao escrito acima.

0 tratamento é realizado com medicações sistêmicas, havendo muitas possibilidades como os derivados sulfamidicos e imidazólicos e a Anfotericina B. O Itraconazol tem sido considerado o fármaco de primeira escolha por apresentar menores taxas de efeitos colaterais e de recidiva (MINISTÉRIO DA SAÚDE, 2013).

O paciente relatado utilizou Anfotericina $B$, em função do comprometimento clínico da criança na ocasião do diagnóstico, sendo esta a droga de escolha em casos graves (MENEZES et al, 2008). Inicialmente, devido ao seu peso (P. $38 \mathrm{Kg}$ ), foi utilizada a dose de adulto, porém o paciente apresentou diversos efeitos colaterais, como náuseas e vômitos, e, por isso, optou-se pela dose infantil, em dias alternados.

O paciente recebeu alta hospitalar e a família se comprometeu a levá-lo, em dias alternados, para receber a medicação intravenosa. Posteriormente, o paciente começaria a receber a medicação oral (Sulfametaxazol - Trimetoprim), reforçando a ideia de que, no acompanhamento ambulatorial, o ideal é que seja feito a longo prazo, uma vez que o tratamento poderá prolongar-se por cerca de um a dois anos. Nesse período, o paciente estará sujeito aos efeitos colaterais das drogas utilizadas, bem como à possibilidade de reativação da doença, frente às condições imunológicas favoráveis (SHIKANAIYASUDA et al., 2006).

\section{CONCLUSÃo}

O conhecimento dos reais valores de casos de paracoccidioidomicose não é fidedigno, pois a mesma não apresenta notificação compulsória. A suspeita dessa enfermidade é facilitada através da associação do caráter endêmico com a história e manifestações clínicas apresentadas pelos pacientes. O relato de caso anteriormente exposto teve por finalidade evidenciar a importância de se elaborar uma anamnese minuciosa, o diagnóstico e o tratamento adequados dessa patologia grave que pode ser fatal. 


\section{REFERÊNCIAS}

ALVES, J. M. A.; DUARTE, M. C.; NEVES, L. A. T.; TOMASCO, E.; LOURENÇO, D.; SANTOS, J. O. S.; YOGUI, L. Levantamento de casos de paracoccidioidomicose infanto-juvenil no HU-UFJF no período de 1992 a 2002. Pediatria Atual, Juiz de Fora, v.17, p. 19-21, 2004.

GONÇALVES, A. J. R.; TERRA, G. M. F.; PASSONI, L. F.; MARTIRE, T.; STAJNBOK, D. C. N.; ENGEL, D. C. Paracoccidioidomicose infanto-juvenil. Relato de dez pacientes recentemente observados na cidade do Rio de Janeiro. Revista Médica do H. S. E. online, Rio de Janeiro, v.34, 2000.

MENEZES, V. M.; SOARES, B. G. O; FONTES, C. J F. Drugs for treating paracoccidioidomycosis. The Cochrane Library, v.4, p. 1-14, 2008.

MINISTÉRIO DA SAÚDE (Brasil). Portal da Saúde: Paracoccidioidomicose. Brasília, 2013. Disponível em: <http://portal.saude.gov.br/portal/saude/profissional/area.cfm?id_area=1662/>. Acesso em 15 mar. 2013.

PALMEIRO, M.; CHERUBINI, K.; YURGEL, L. S. Paracoccidioidomicose-Revisão da Literatura. Scientia Medica, Porto Alegre, v. 15, 2005.

SHIKANAI- YASUDA, M. A.; FILHO, F. Q. T.; MENDES, R. P.; COLOMBO, A. L.; MORETTI, M. L. Consenso em paracoccidioidomicose. Revista as Sociedade Brasileira de Medicina Tropical, Uberaba, v.39, p. 297-310, 2006.

SZTAJNBOK, B. C. N.; VASCONCELOS, M. M.; LIBERAL, E. F. P. Paracoccidioidomicose. In: SAMPAIO, M. G.; ABREU, T. F. Infectologia: Pediatria - Soperj. 1. ed. Rio de Janeiro: Rio de Janeiro, 2012.

TAVARES, W.; MARINHO, L. A. C. Paracoccidioidomicose. In: MENDES, R. P.; REIS, V. L. L; TAVARES, W. Rotinas de diagnóstico e tratamento das doenças infecciosas e parasitárias. 3.ed. São Paulo: São Paulo, 2012. p. 810-825.

TELLES, F. Boletim Epidemiológico da Secretaria de Estado de Saúde do Paraná, Curitiba, 2001. Disponível em <vigiepi/boletim/inverno-2001 >. Acesso em: 15 mar. 2013.

TOLEDO, G. L.; MARZOLA, C.; FILHO, J, L, T.; CAPELARI, M, M. Blastomicose sul americana- apresentação de caso clínico tratado com associação de inidazóis sistêmico e tópico. Revista Portuguesa de Estomatologia, Medicina Dentária e Cirurgia Maxilofacial, Portugal, v. 52, p.83-88, 2011.

TONELLI, E.; FREIRE, L. M. S. Micoses profundas II - Paracoccidioidomicose. In: ANDRADE, G. M. Q.; NOGUEIRA, M. G. S. Doenças infecciosas na infância e adolescência. 2. ed. São Paulo: São Paulo, 2000. p. $1490-1515$. 